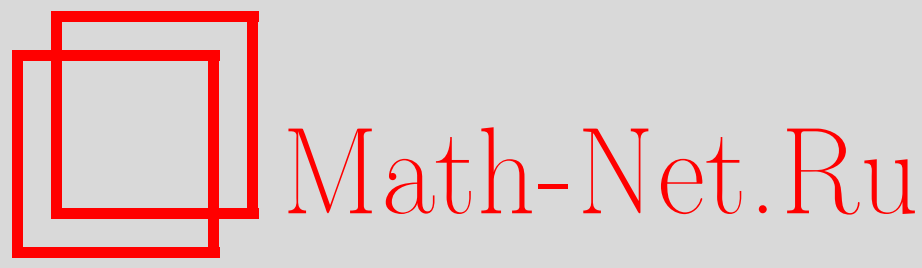

В. Н. Лихачев, Г. А. Виноградов, Интегральные характеристики волновых пакетов в задаче об эволюции волновой функции на одномерной решетке, ТМФ, 2018, том 197, номер 2, 257-268

DOI: https://doi.org/10.4213/tmf9496

Использование Общероссийского математического портала Math-Net.Ru подразумевает, что вы прочитали и согласны с пользовательским соглашением http://www . mathnet.ru/rus/agreement

Параметры загрузки:

IP : 3.81 .55 .215

26 апреля 2023 г., 06:05:29

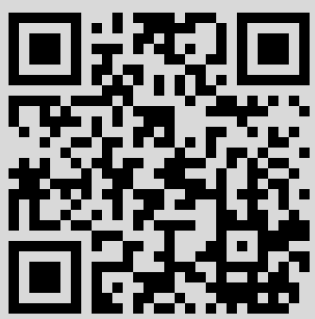




\title{
ИНТЕГРАЛЬНЫЕ ХАРАКТЕРИСТИКИ ВОЛНОВЫХ ПАКЕТОВ В ЗАДАЧЕ ОБ ЭВОЛЮЦИИ ВОЛНОВОЙ ФУНКЦИИ НА ОДНОМЕРНОЙ РЕШЕТКЕ
}

\begin{abstract}
Рассмотрена квантовая динамика переноса заряда на решетке в приближении сильной связи. Аналитически вычисляются интегральные характеристики распространяющегося по решетке волнового пакета. Основное внимание уделено вычислению среднего и среднеквадратичного смещений. Также получены выражения для моментов более высоких порядков, которые выражаются в виде рядов для квадратов функций Бесселя, что может представлять самостоятельный интерес.
\end{abstract}

Ключевые слова: квантовая динамика, приближение сильной связи, моменты функции распределения.

DOI: https://doi.org/10.4213/tmf9496

\section{1. ВВЕДЕНИЕ}

Недавно в ряде публикаций было экспериментально установлено [1]-[4], что заряд, тем или иным образом инжектированный в регулярную решетку, обеспечивает электропроводность довольно необычным образом. Одномерные регулярные решетки синтезировали из одинаковых пар оснований ДНК, чаще всего использовали аденин-тимин. Таким образом получали олигомеры $(\mathrm{A}: \mathrm{T})_{N}$ с длиной цепи $N$ до 100 пар повторяющихся оснований. В этих и ряде других работ было показано, что, во-первых, перенос заряда происходит очень быстро, во-вторых, осуществляется "баллистически", т.е. скорость движения заряда постоянна и не зависит от размеров решетки, и, в-третьих, вероятность переноса заряда также практически не зависит от длины цепочки в пределах нескольких десятков постоянной решетки ДНК. Из двух наиболее вероятных механизмов переноса заряда - поляронного и квантово-динамического - предпочтение отдается последнему. Причина в том, что волновая функция заряда очень быстро распространяется по цепи и заряд за счет электрон-фононного взаимодействия не успевает деформировать решетку для локализации и формирования полярона (характерное квантовое время перескока

* Институт биохимической физики им. Н. М. Эмануэля РАН, Москва, Россия. E-mail: gvin@deom.chph.ras.ru 
на два порядка меньше характерного колебательного времени решетки). Аналогичные результаты были получены для одномерных регулярных цепочек полипептидов. Авторы цитированных работ, рассматривая лишь перескоковый механизм проводимости и туннелирование, не смогли объяснить полученные ими результаты.

В настоящей работе мы изучаем квантовый механизм электропроводимости, когда волновая функция заряда формирует движущийся локализованный волновой пакет, который и обеспечивает перенос. Ранее подобный механизм был рассмотрен для проводимости [5], [6] и переноса квантовых колебательных возбуждений [7], [8].

В задачах о распространении квантовых возбуждений на решетке часто используют первый и второй моменты волнового пакета в момент времени $t$. Эти моменты определяются так: $M_{1}(t)=\sum_{m} m|\Psi(m ; t)|^{2}$ и $M_{2}(t)=\sum_{m} m^{2} \mid \Psi\left(m ;\left.t\right|^{2}\right.$, где $\Psi(m ; t)-$ волновая функция квантового возбуждения на узле $m$ решетки. Момент $M_{1}(t)$ определяет "центр тяжести" распространяющегося волнового пакета, а $M_{2}(t)$ - интегральную форму (размер) пакета.

Первый и второй моменты очень часто используются в физике твердого тела в задачах о переносе энергии и заряда на решетках с дефектами и примесями. В особенности это относится к изучению характеристик волнового пакета при андерсоновской локализации.

Второй момент позволяет различить режимы диффузии и локализации заряда [9] по обе стороны от точки перехода металл-диэлектрик. При диффузии второй момент растет по степенно́му закону от времени $\sim t^{\alpha}$ и показатель степени меняется в довольно широких пределах [10]-[13]. В некоторых случаях удалось выявить связь второго момента с мультифрактальными свойствами собственных значений гамильтониана [14], [15]. Похоже, что высшие ( $k$-е) моменты меняются со временем $M_{k}(t) \sim t^{k \beta}$ с показателем степени, пропорциональным $k[16]$.

До сих пор моменты плотности волновой функции вычислялись численно при решении эволюционного уравнения Шредингера. Интересно получить точные аналитические выражения для этих моментов, поскольку это позволит понять зависимость полученных значений от параметров задачи. Именно решению этого вопроса и посвящена настоящая работа.

\section{2. ПОСТАНОВКА ЗАДАЧИ}

Рассмотрим квантовую динамику движения электрона на одномерной решетке. Рассмотрение проводится в рамках приближения сильной связи, иначе называемого приближением Хюккеля. В этой постановке задача имеет два параметра: интеграл перескока и энергия волновой функции на узле. Значение интеграла перескока можно произвольно выбрать равным - 1, что одновременно означает выбор масштаба энергии. Энергия связи волновой функции с узлами решетки равна +2 . Такое значение выбрано для того, чтобы дно валентной зоны соответствовало нулевому значению энергии. Решетка однородна и электрон в начальный момент времени находится на одном из ее узлов. Решается нестационарная задача об эволюции волновой функции заряда на решетке.

Гамильтониан рассматриваемой задачи во вторичном квантовании имеет вид

$$
H=\sum_{m} c_{m}^{+}\left(2 c_{m}^{-}-c_{m-1}^{-}-c_{m+1}^{-}\right),
$$

где $m$ - номер узла решетки. 
Решается уравнение Шредингера для волновой функции $\Psi(m ; t)$. В качестве начального условия выбирается волновая функция электрона, локализованного на произвольном узле решетки. Будут рассмотрены два случая - бесконечной и полубесконечной решетки.

\section{3. КВАНТОВАЯ ДИНАМИКА ЭЛЕКТРОНА В ОДНОРОДНОЙ РЕШЕТКЕ}

Сначала рассмотрим задачу на бесконечной решетке. Найдем решение временно́го уравнения Шредингера при условии, что в начальный момент времени электрон находится в начале координат, т. е. $\Psi_{1}(0 ; t=0)=1$. В силу трансляционной инвариантности бесконечной решетки такое начальное условие носит общий характер. Задача решается с помощью стандартной процедуры разложения по собственным функциям гамильтониана (1).

Собственные функции $\phi_{k}(m)$ и собственные значения энергии $E(k)$ гамильтониана (1) хорошо известны:

$$
\phi_{k}(m)=\frac{1}{\sqrt{2 \pi}} e^{i k m}, \quad E(k)=2(1-\cos k), \quad|k|<\pi .
$$

Решение временно́го уравнения для бесконечной решетки выражается через собственные функции стандартным образом:

$$
\Psi_{1}(m ; t)=\int_{-\pi}^{\pi} d k \phi_{k}(m) \phi_{k}(0) e^{-E(k) t} .
$$

Вычисляя этот интеграл, получаем

$$
\Psi_{1}(m ; t)=\mathrm{J}_{m}(2 t) e^{-2 i t+i \pi m / 2},
$$

где $\mathrm{J}_{m}$ - функция Бесселя порядка $m$.

На рис. 1 показана плотность волновой функции $\left|\Psi_{1}(m ; t)\right|^{2}$ в момент времени $t=10$. Волновая функция представляет собой два разбегающихся в разные стороны волновых пакета. Передние фронты пакетов двигаются с максимально возможными групповыми скоростями $v= \pm 2$.

Теперь рассмотрим случай полубесконечной решетки $(m=1,2, \ldots)$. Пусть в начальный момент электрон находится в узле $m_{0}: \Psi_{2}\left(m, m_{0} ; t=0\right)=\delta_{m, m_{0}}$. Собственные функции $\phi_{k}(m)$ и собственные значения $E(k)$ этой задачи также известны:

$$
\phi_{k}(m)=\sqrt{\frac{2}{\pi}} \sin [k(m+1)], \quad E(k)=2(1-\cos k), \quad 0<k<\pi .
$$

Решение временно́го уравнения выражается через собственные функции известным способом:

$$
\Psi_{2}\left(m, m_{0} ; t\right)=\frac{2}{\pi} \int_{-\pi}^{\pi} d k \sin [k(m+1)] \sin \left[k\left(m_{0}+1\right)\right] e^{-i E(k) t} .
$$

Вычисляя этот интеграл, получаем

$$
\begin{aligned}
\Psi_{2}\left(m, m_{0} ; t\right)=e^{-2 i t}[\exp ( & \left.i \frac{\pi}{2}\left|m-m_{0}\right| \mathrm{J}_{\left|m-m_{0}\right|}(2 t)\right)+ \\
& \left.+\exp \left(i \frac{\pi}{2}\left(m+m_{0}+2\right) \mathrm{J}_{m+m_{0}+2}(2 t)\right)\right] .
\end{aligned}
$$




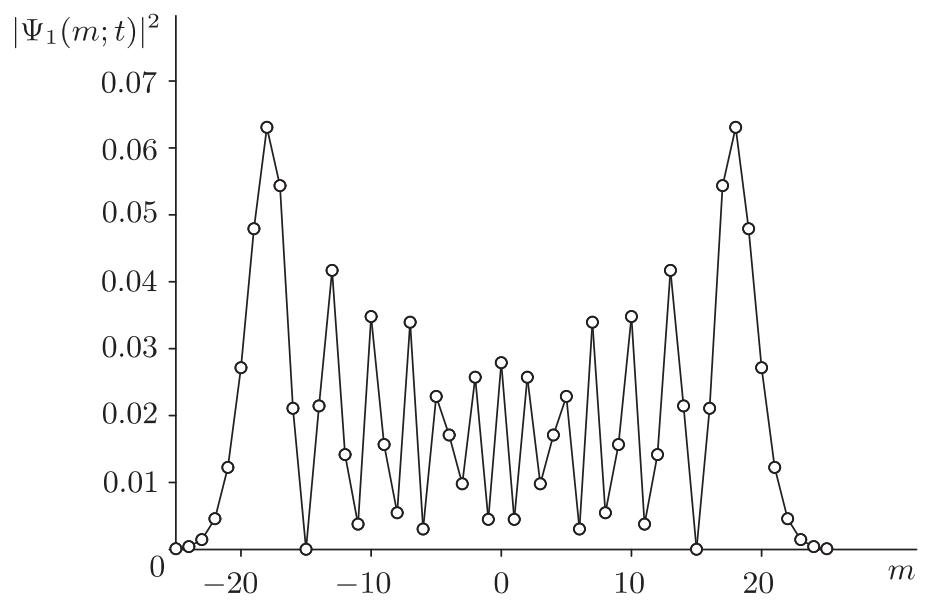

Рис. 1. Плотность волновой функции $|\Psi(m ; t)|^{2}$ в момент времени $t=10$.

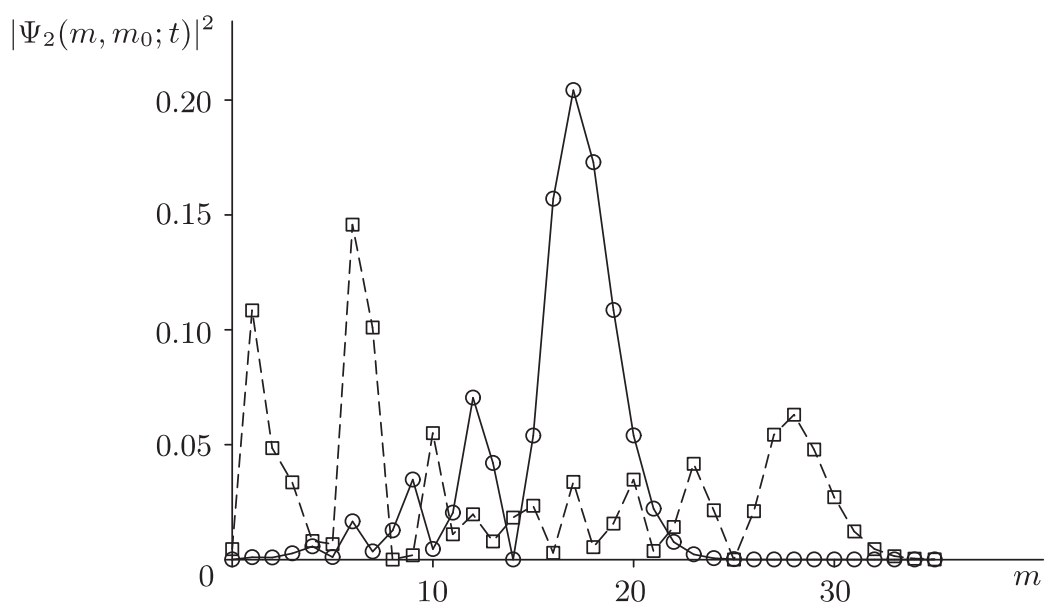

Рис. 2. Плотность волновой функции $\left|\Psi_{2}\left(m, m_{0} ; t\right)\right|^{2}$ в момент времени $t=10$ для разных начальных условий. Сплошная линия и кружки: $m_{0}=1$, штриховая линия и квадраты: $m_{0}=10$. В случае $m_{0}=10$ у импульса есть две составляющие: один импульс непосредственно идет направо (максимум на узле $m=28)$, другой импульс первоначально идет налево и затем отражается от начала цепочки (максимум на узле $m=6$ ).

На рис. 2 приведен график функции $\left|\Psi_{2}\left(m, m_{0} ; t\right)\right|$ в момент времени $t=10$ для разных начальных условий.

Далее мы вычислим "интегральные" характеристики волновых пакетов функций $\Psi_{1}(m ; t)$ и $\Psi_{2}\left(m, m_{0} ; t\right)$, а именно получим выражения для зависимости от времени средней координаты и "размера" пакета. Предварительно получим выражения для моментов распределения, задаваемых квадратами функций Бесселя. 


\section{4. МОМЕНТЫ ФУНКЦИИ РАСПРЕДЕЛЕНИЯ, ЗАДАВАЕМЫЕ КВАДРАТАМИ ФУНКЦИЙ БЕССЕЛЯ}

Рассмотрим сначала предварительную задачу о вычислении следующих моментов произвольного порядка $k$ :

$$
\mathcal{M}_{k}(x) \equiv \sum_{n=1}^{\infty} n^{k} \mathrm{~J}_{n}^{2}(x)
$$

где переменная $x$ заменяет для краткости использованную выше переменную $2 t$.

Рассмотрим нулевой момент $\mathcal{M}_{0}(x)$. Из условия нормировки на единицу волновой функции $\Psi_{1}(m ; t)$ получаем

$$
\mathcal{M}_{0}(x) \equiv \sum_{n=1}^{\infty} \mathrm{J}_{n}^{2}(x)=\frac{1}{2}\left[1-\mathrm{J}_{0}^{2}(x)\right]
$$

Заметим, что это равенство приведено в монографии [17].

Получим дифференциальные соотношения, связывающие моменты разных порядков. Для этого применим два рекуррентных соотношения для функций Бесселя:

$$
\frac{x}{2}\left[\mathrm{~J}_{n-1}(x)+\mathrm{J}_{n+1}(x)\right]=n \mathrm{~J}_{n}(x), \quad\left[\mathrm{J}_{n-1}(x)-\mathrm{J}_{n+1}(x)\right]=2 \frac{d \mathrm{~J}_{n}(x)}{d x} .
$$

Перемножая эти соотношения, получим

$$
\frac{x}{2}\left(\mathrm{~J}_{0}^{2}(x)-\mathrm{J}_{n+1}^{2}(x)\right)=n \frac{d \mathrm{~J}_{n}^{2}(x)}{d x} .
$$

Умножая обе части уравнения (10) на $n^{k}$ и суммируя по $n$, приходим к уравнению

$$
\frac{x}{2}\left(\mathrm{~J}_{0}^{2}(x)+\sum_{n=1}^{\infty} \mathrm{J}_{n}^{2}(x)\left[(n+1)^{2}-(n-1)^{2}\right]\right)=\frac{d \mathcal{M}_{n+1}(x)}{d x}
$$

Записывая это равенство через моменты, получим

$$
\frac{d \mathcal{M}_{n+1}(x)}{d x}=\frac{x}{2}\left[J_{0}^{2}(x)+2 \sum_{k=1}^{[(k+1) / 2]} C_{k}^{2 m-1} \mathcal{M}_{k+1-2 m}\right], \quad \mathcal{M}_{n}(0)=0
$$

Уравнения (12) позволяют выразить производную четного момента через четные моменты меньших порядков (то же будет справедливо и для нечетных моментов). Выпишем несколько первых уравнений для четных моментов:

$$
\begin{aligned}
& \frac{d \mathcal{M}_{2}(x)}{d x}=\frac{x}{2}\left[\mathrm{~J}_{0}^{2}(x)+2 \mathcal{M}_{0}(x)\right] \\
& \frac{d \mathcal{M}_{4}(x)}{d x}=\frac{x}{2} \mathrm{~J}_{0}^{2}(x)+x\left[\mathcal{M}_{0}(x)+3 \mathcal{M}_{2}(x)\right] \\
& \frac{d \mathcal{M}_{6}(x)}{d x}=\frac{x}{2} \mathrm{~J}_{0}^{2}(x)+x\left[\mathcal{M}_{0}(x)+10 \mathcal{M}_{2}(x)+5 \mathcal{M}_{4}(x)\right]
\end{aligned}
$$


Поскольку $\mathrm{J}_{0}^{2}(x)+2 \mathcal{M}_{0}(x)=1$ (см. (9)), то уравнения (13) для четных моментов можно переписать в более простом виде:

$$
\begin{aligned}
& \frac{d \mathcal{M}_{2}(x)}{d x}=\frac{x}{2} \\
& \frac{d \mathcal{M}_{4}(x)}{d x}=\frac{x}{2}+3 x \mathcal{M}_{2}(x) \\
& \frac{d \mathcal{M}_{6}(x)}{d x}=\frac{x}{2}+5 x\left[2 \mathcal{M}_{2}(x)+\mathcal{M}_{4}(x)\right] .
\end{aligned}
$$

Непосредственным интегрированием получаем, что четные моменты $\mathcal{M}_{k}$ суть полиномы четных степеней по $k$ :

$$
\begin{aligned}
& \mathcal{M}_{2}(x)=\frac{x^{2}}{4} \\
& \mathcal{M}_{4}(x)=\frac{1}{4} x^{2}+\frac{3}{16} x^{4} \\
& \mathcal{M}_{6}(x)=\frac{1}{4} x^{2}+\frac{5}{32} x^{4}\left(x^{2}+6\right) .
\end{aligned}
$$

Заметим, что свободный член отсутствует в силу условия $\mathcal{M}_{k}(0)=0$. Отметим, что выражение для второго момента $\mathcal{M}_{2}(x)$ также приведено в [17].

Выпишем теперь уравнения (12) для нечетных моментов:

$$
\begin{aligned}
& \frac{d \mathcal{M}_{1}(x)}{d x}=\frac{x}{2}\left[\mathrm{~J}_{0}^{2}(x)+\mathrm{J}_{1}^{2}(x)\right] \\
& \frac{d \mathcal{M}_{3}(x)}{d x}=\frac{x}{2} \mathrm{~J}_{0}^{2}(x)+4 x\left[\mathcal{M}_{1}(x)+\mathcal{M}_{3}(x)\right] \\
& \frac{d \mathcal{M}_{5}(x)}{d x}=\frac{x}{2} \mathrm{~J}_{0}^{2}(x)+2 x\left[3 \mathcal{M}_{1}(x)+10 \mathcal{M}_{3}(x)+3 \mathcal{M}_{5}(x)\right]
\end{aligned}
$$

Последовательным интегрированием получаем следующие аналитические выражения для нечетных моментов (подробности вычисления интегралов см. в приложении; там же показано, что получение каждого следующего нечетного момента требует лишь алгебраических вычислений):

$$
\begin{aligned}
\mathcal{M}_{1}(x)= & \frac{1}{2}\left\{x^{2}\left[\mathrm{~J}_{0}^{2}(x)+\mathrm{J}_{1}^{2}(x)\right]-x \mathrm{~J}_{0}(x) \mathrm{J}_{1}(x)\right\}, \\
\mathcal{M}_{3}(x)= & \frac{1}{3}\left\{x^{4}\left[\mathrm{~J}_{0}^{2}(x)+\mathrm{J}_{1}^{2}(x)\right]-x^{3} \mathrm{~J}_{0}(x) \mathrm{J}_{1}(x)\right\}+\frac{x^{2}}{12}\left[3 \mathrm{~J}_{0}^{2}(x)+\mathrm{J}_{1}^{2}(x)\right], \\
\mathcal{M}_{5}(x)= & \frac{4}{15}\left\{x^{6}\left[\mathrm{~J}_{0}^{2}(x)+\mathrm{J}_{1}^{2}(x)\right]-x^{5} \mathrm{~J}_{0}(x) \mathrm{J}_{1}(x)\right\}+ \\
& +\frac{2 x^{4}}{15}\left[7 \mathrm{~J}_{0}^{2}(x)+6 \mathrm{~J}_{1}^{2}(x)\right]-\frac{11 x^{3}}{15} \mathrm{~J}_{0}(x) \mathrm{J}_{1}(x)+\frac{x^{2}}{4}\left[\mathrm{~J}_{0}^{2}(x)-\frac{1}{15} \mathrm{~J}_{1}^{2}(x)\right] .
\end{aligned}
$$

Для проверки этих формул можно использовать две производные функций Бесселя: $d \mathrm{~J}_{0} / d x=-\mathrm{J}_{1}$ и $d \mathrm{~J}_{1} / d x=\mathrm{J}_{0}-\mathrm{J}_{1} / x$. 


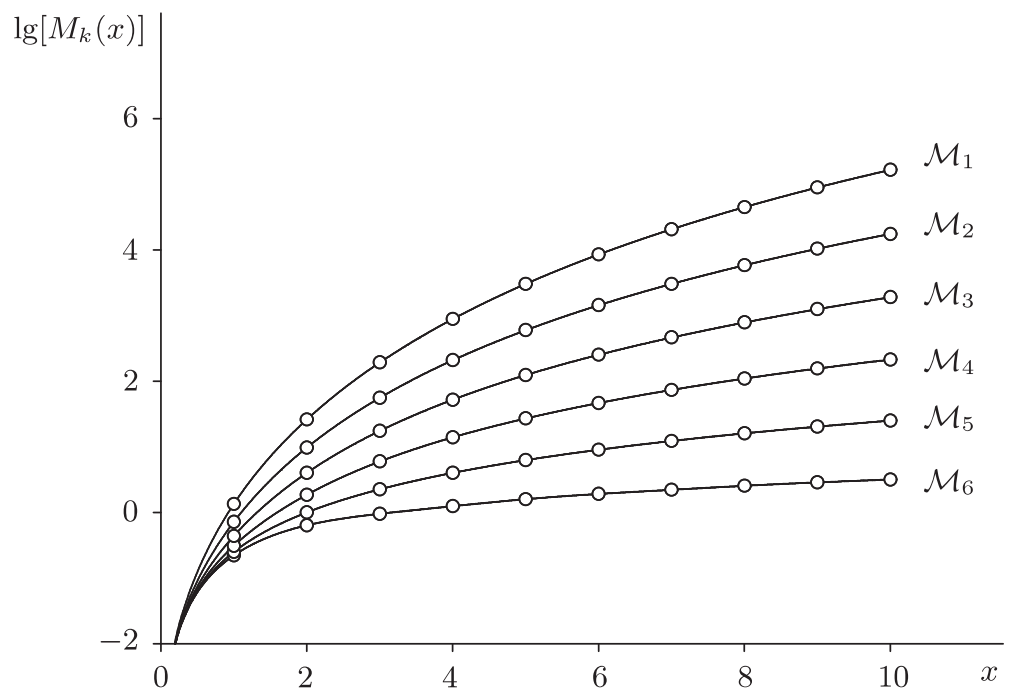

Рис. 3. Моменты $\mathcal{M}_{1}-\mathcal{M}_{6}$. Сплошные линии - вычисления по формулам (15) и (17). Кружки - численное суммирование соответствующих рядов. Разница по порядку величины составляет $10^{-15}$.

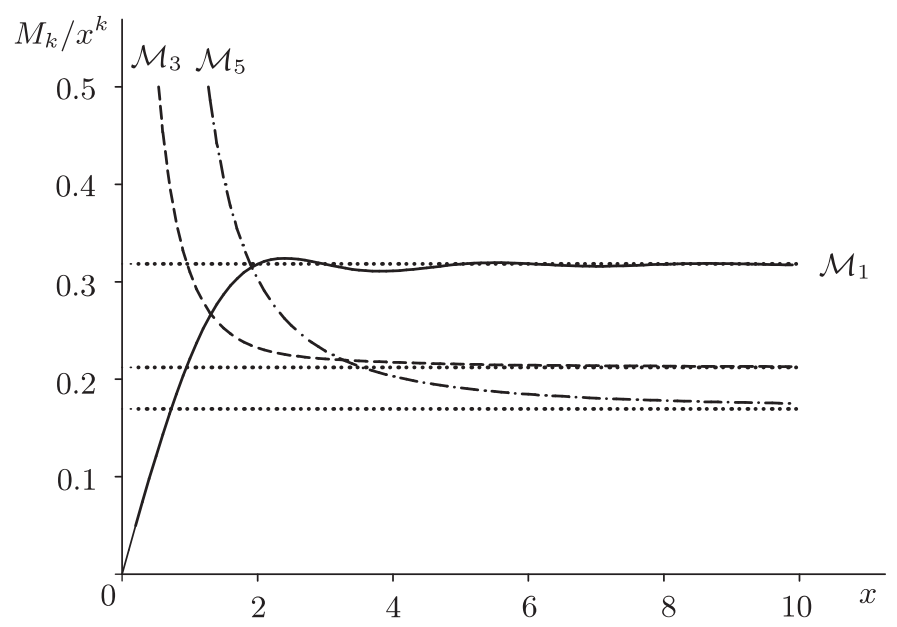

Рис. 4. Асимптотическое поведение моментов $\mathcal{M}_{1}$ (сплошная линия), $\mathcal{M}_{3}$ (штриховая линия) и $\mathcal{M}_{5}$ (штрихпунктирная линия). Предельные значения для $\mathcal{M}_{1} / x, \mathcal{M}_{3} / x^{3}$ и $\mathcal{M}_{5} / x^{5}$ в соответствии с формулой $(20)$ равны $1 / \pi, 2 / 3 \pi$ и $8 / 15 \pi$ соответственно (эти асимптотические значения показаны пунктирными линиями).

Отметим, что выражение для первого момента $\mathcal{M}_{1}(x)$ можно найти в [17], где отдельно приведены выражения для сумм четных и нечетных членов суммы $\mathcal{M}_{1}$. На рис. 3 показано поведение моментов $\mathcal{M}_{1}-\mathcal{M}_{6}$. 
Приведем асимптотические формулы для больших значений $x$. Для четных моментов имеем

$$
\mathcal{M}_{k}(x) \sim \frac{1}{2} \frac{(k-1) ! !}{k ! !} x^{k}, \quad x \gg 1
$$

где $k$ - четное число.

Для нечетных моментов в приложении приведено выражение для $\mathcal{M}_{k}(x)$ в случае $x \gg 1$. Используя асимптотику функций Бесселя $\mathrm{J}_{0}^{2}(x)+\mathrm{J}_{1}^{2}(x) \sim 2 / \pi x$, имеем

$$
\mathcal{M}_{k}(x) \sim \frac{1}{\pi} \frac{(k-1) ! !}{k ! !}, \quad x \gg 1
$$

где $k$ нечетно. На рис. 4 показано асимптотическое поведение моментов $\mathcal{M}_{1}, \mathcal{M}_{3}, \mathcal{M}_{5}$.

Приведем выражение для высоких $(k \gg 1)$ моментов. Преобразуем асимптотики (18), (19) по формулам Стирлинга с учетом поправочного множителя $(1+1 / 12 x)$. Оказывается, что асимптотики для четных и нечетных моментов описываются единой формулой:

$$
\mathcal{M}_{k}(x) \sim \frac{x^{k}}{\sqrt{\pi(2 k+1)}}, \quad x \gg 1, \quad k \gg 1
$$

\section{5. ПЕРВЫЙ И ВТОРОЙ МОМЕНТЫ ВОЛНОВЫХ ПАКЕТОВ $\Psi_{1}(m ; t)$ И $\Psi_{2}(m, 1 ; t)$}

Найдем зависимость от времени средней и среднеквадратичной координат волнового пакета $\Psi_{1}(m ; t)$ (см. $\left.(4)\right)$. Для бо́льшей наглядности рассмотрим только "половину" волнового пакета, бегущую, например, направо.

Среднюю координату и средний квадрат координаты пакета определим следующим образом:

$$
\begin{aligned}
\langle m\rangle & \equiv \frac{\sum_{m=1}^{\infty} m|\Psi(m ; t)|^{2}}{\sum_{m=1}^{\infty}|\Psi(m ; t)|^{2}}, \\
\left\langle m^{2}\right\rangle & \equiv \frac{\sum_{m=1}^{\infty} m^{2}|\Psi(m ; t)|^{2}}{\sum_{m=1}^{\infty}|\Psi(m ; t)|^{2}} .
\end{aligned}
$$

Волновая функция $\Psi_{1}(m ; t)$ с точностью до фазового множителя совпадает с $\mathrm{J}_{m}(2 t)$ (см. (4)). Поэтому среднее $\langle m\rangle$ есть отношение моментов $\mathcal{M}_{1}(2 t) / \mathcal{M}_{0}(2 t)\left(\mathcal{M}_{0}\right.$ см. (9)):

$$
\langle m\rangle=\frac{2 \mathcal{M}_{1}(2 t)}{1-\mathrm{J}_{0}^{2}(2 t)}
$$

Аналогично для среднего квадрата получаем $\left\langle m^{2}\right\rangle=\mathcal{M}_{2}(2 t) / \mathcal{M}_{0}(2 t)$. Так как $\mathcal{M}_{2}(2 t)=t^{2}$, имеем

$$
\left\langle m^{2}\right\rangle=\frac{2 t^{2}}{1-\mathrm{J}_{0}^{2}(2 t)}
$$




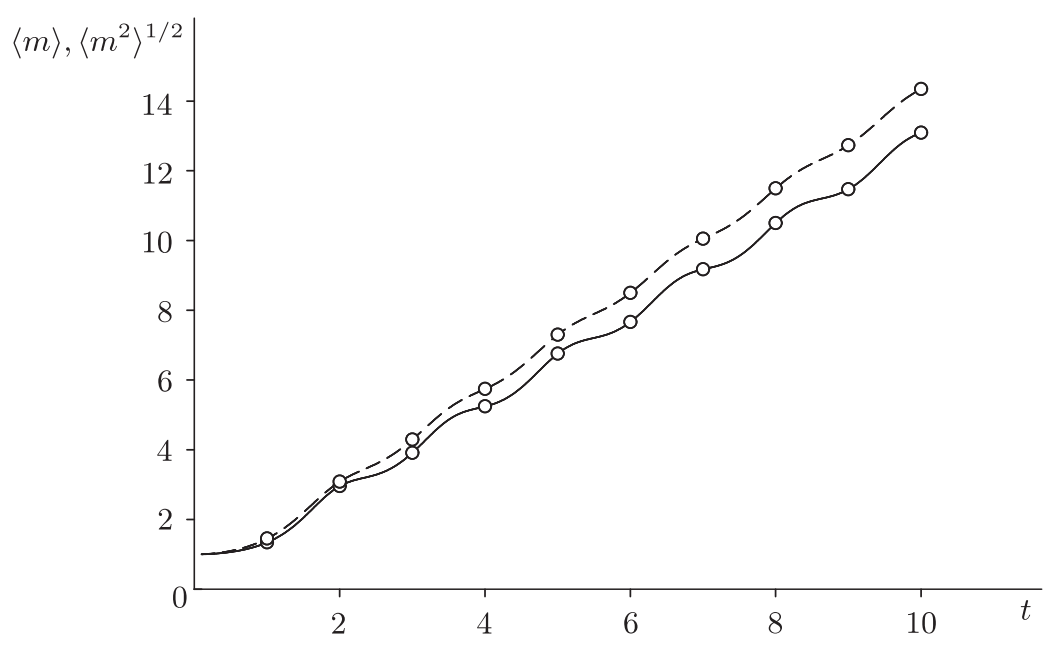

Рис. 5. Средняя (сплошная линия) и среднеквадратичная (штриховая линия) координаты волнового пакета $\Psi_{1}(m ; t)$, вычисленные по формулам (22), (23). Кружки - прямое суммирование численного решения уравнения Шредингера. Разница по порядку величины составляет $10^{-10}$.

На рис. 5 показана зависимость от времени средней и среднеквадратичной координат пакета в зависимости от времени.

При $t \gg 1$ имеем следующие асимптотики: $\langle m\rangle \sim 4 t / \pi$ и $\left\langle m^{2}\right\rangle \sim 2 t^{2}$. Размер (ширина) пакета растет как $0.6 t$.

Рассмотрим теперь волновой пакет $\Psi_{2}\left(m, m_{0} ;, t\right)$ на полубесконечной решетке (см. (7)) для случая $m_{0}=0$. В этом случае, используя рекуррентные соотношения для функций Бесселя, для функции $\Psi_{2}$ получаем

$$
\Psi_{2}(m, 0 ; t)=e^{-2 i t+i \pi m / 2} \frac{m+1}{t} \mathrm{~J}_{m+1}(2 t) .
$$

Для волнового пакета $\Psi_{2}(m, 0 ; t)$ определим средние $\langle m\rangle$ и $\left\langle m^{2}\right\rangle$ прежними формулами (21). Получаем следующие выражения для этих средних через моменты $M_{k}(2 t)$ :

$$
\langle m\rangle=\frac{\mathcal{M}_{3}(2 t)-\mathcal{M}_{2}(2 t)}{t^{2}-\mathrm{J}_{1}^{2}(2 t)}, \quad\left\langle m^{2}\right\rangle=\frac{\mathcal{M}_{4}(2 t)-2 \mathcal{M}_{3}(2 t)+\mathcal{M}_{2}(2 t)}{t^{2}-J_{1}^{2}(2 t)} .
$$

На рис. 6 показана зависимость от времени средней и среднеквадратичной координат пакета $\Psi_{2}(m, 0 ; t)$ в зависимости от времени. Локализация волнового пакета выглядит весьма эффектно в двумерном случае для квадранта, когда сначала электрон находится в начале координат. Волновая функция в этом случае, очевидно, является произведением $\Phi(m, n ; t)=\Psi_{2}(m, 0 ; t) \Psi_{2}(n, 0 ; t)$. Пакет движется по диагонали со скоростью $\{16 / 3 \pi, 16 / 3 \pi\}$; размер пакета составляет $\sim\{0.3 t \cdot 03 t\}($ см. рис. 7).

В заключение кратко суммируем полученные результаты. В настоящей работе в рамках приближения сильной связи проанализирована возможность переноса заряда посредством волнового пакета. Вычисления первого момента показали, 


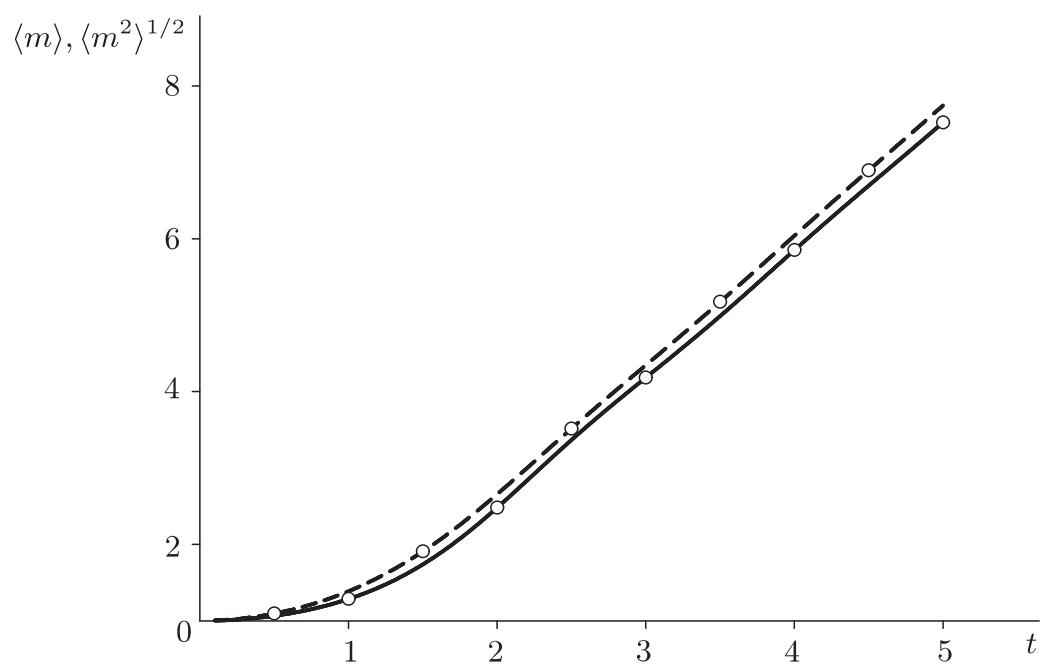

Рис. 6. Средняя (сплошная линия) и среднеквадратичная (штриховая линия) координаты волнового пакета $\Psi_{2}(m, 0 ; t)$, вычисленные по формулам (25). Кружки - прямое суммирование численного решения уравнения Шредингера. Разница по порядку величины составляет $10^{-10}$.

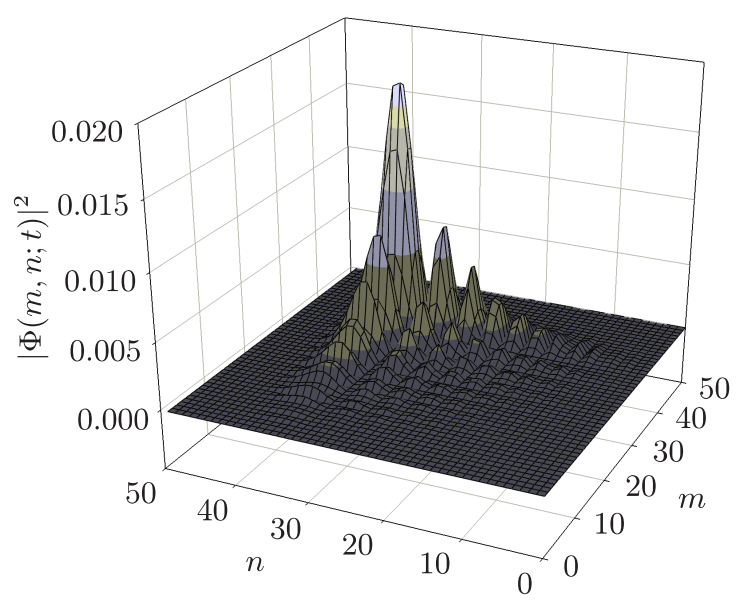

Рис. 7. Распространяющийся в квадранте волновой пакет в момент $t=20$.

что волновой пакет движется с постоянной скоростью. Его ширина, определяемая вторым моментом, относительно медленно растет со временем. Таким образом, на длине решетки в несколько десятков постоянных решетки волновой пакет сохраняет свою компактную форму. Эти результаты косвенно подтверждают экспериментальные результаты [1]-[4]. Дополнительно вычислены ряды для квадратов функций Бесселя, что может представлять самостоятельный интерес. 
ПРИЛОЖЕНИЕ

Рассмотрим получение нечетных моментов. Исходными являются следующие интегралы:

$$
\begin{aligned}
& \int x \mathrm{~J}_{0}^{2}(x) d x=\frac{x^{2}}{2}\left[\mathrm{~J}_{0}^{2}(x)+\mathrm{J}_{1}^{2}(x)\right], \\
& \int x \mathrm{~J}_{1}^{2}(x) d x=\frac{x^{2}}{2}\left[\mathrm{~J}_{0}^{2}(x)+\mathrm{J}_{1}^{2}(x)\right]-x \mathrm{~J}_{0}(x) \mathrm{J}_{1}(x), \\
& \int x^{2} \mathrm{~J}_{0}(x) \mathrm{J}_{1}(x) d x=\frac{x^{2}}{2} \mathrm{~J}_{1}^{2}(x) .
\end{aligned}
$$

Мы покажем, что все последующие (высшие по степеням $x$ ) интегралы сводятся к этим трем.

Рассмотрим уравнение для первого момента:

$$
\frac{d \mathcal{M}_{1}(x)}{d x}=\frac{x}{2}\left[\mathrm{~J}_{0}^{2}(x)+\mathrm{J}_{1}^{2}(x)\right]
$$

Пользуясь соотношениями (26), получаем

$$
\mathcal{M}_{1}(x)=\frac{x^{2}}{2}\left[\mathrm{~J}_{0}^{2}(x)+\mathrm{J}_{1}^{2}(x)\right]-\frac{x}{2} .
$$

Общая структура нечетного момента $\mathcal{M}_{k}(x)$ имеет следующий вид:

$$
\mathcal{M}_{k}(x)=P_{0}^{k+1}(x) \mathrm{J}_{0}^{2}(x)+P_{1}^{k+1}(x) \mathrm{J}_{1}^{2}(x)+Q^{k}(x) \mathrm{J}_{0}(x) \mathrm{J}_{1}(x),
$$

где $P_{0}$ и $P_{1}$ - четные полиномы степени $k+1, Q$ - нечетный полином степени $k$.

Уравнение для следующего момента $\mathcal{M}_{k+2}(x)$ имеет вид

$$
\frac{d \mathcal{M}_{k+2}(x)}{d x}=\frac{x}{2} \mathrm{~J}_{0}^{2}(x)+x \cdot Z,
$$

где $Z$ есть линейная комбинация нечетных моментов $\mathcal{M}_{1}, \mathcal{M}_{3}, \ldots, \mathcal{M}_{k}$.

Таким образом, для вычисления момента $\mathcal{M}_{k+2}(x)$ необходимо уметь вычислять следующие интегралы:

$$
\begin{gathered}
I_{0}^{(m)} \equiv \int x^{m} \mathrm{~J}_{0}^{2}(x) d x, \quad I_{1}^{(m)} \equiv \int x^{m} \mathrm{~J}_{1}^{2}(x) d x \\
Q^{(m)} \equiv \int x^{m} \mathrm{~J}_{0}(x) \mathrm{J}_{1}(x) d x
\end{gathered}
$$

Но эти интегралы могут быть сведены к таким же, но со степенью $m-2$, и в конечном итоге вычисление сведется к трем интегралам (26). Это сведе́ние осуществляется с помощью следующих легко проверяемых рекуррентных соотношений:

$$
\begin{gathered}
(m+1) I_{0}^{(m)}(x)+(m-1) I_{1}^{(m)}(x)=x^{m+1}\left[\mathrm{~J}_{0}^{2}(x)+\mathrm{J}_{1}^{2}(x)\right], \\
I_{0}^{(m)}(x)-I_{1}^{(m)}(x)=x^{m} \mathrm{~J}_{0}(x) \mathrm{J}_{1}(x)-(m-1) Q^{(m-1)}(x), \\
Q^{(m)}(x)=-\frac{x^{m}}{2} \mathrm{~J}_{0}^{2}(x)+\frac{m}{2} I_{0}^{(m-1)}(x) .
\end{gathered}
$$


Заметим, что с помощью уравнений (31) можно получить выражение для первых двух ведущих членов момента $M_{k}$ :

$$
\mathcal{M}_{k}(x) \sim \frac{(k-1) ! !}{2 \cdot k ! !}\left\{x\left[\mathrm{~J}_{0}^{2}(x)+\mathrm{J}_{1}^{2}(x)\right]-x^{k} \mathrm{~J}_{0}(x) \mathrm{J}_{1}(x)\right\}
$$

\section{Список литературы}

[1] K. E. Augustyn, J. C. Genereux, J.K. Barton, "Distance-independent DNA charge transport across an adenine tract", Angew. Chem. Internat. Ed., 46 (2007), 5731-5733.

[2] B. Elias, J. C. Genereux, J. K. Barton, "Ping-pong electron transfer through DNA", Angew. Chem. Internat. Ed., 47 (2008), 9067-9070.

[3] J. C. Genereux, J. K. Barton, "Mechanisms for DNA charge transport", Chem. Rev., 110:3 (2010), 1642-1662.

[4] K. Kawai, H. Kodera, Y. Osakada, T. Majima, "Sequence-independent and rapid long-range charge transfer through DNA", Nature Chem., 1 (2009), 156-159.

[5] В.Н. Лихачев, Т. Ю. Астахова, Г. А. Виноградов, “'Электронный пинг-понг' на одномерной решетке. Движение волнового пакета до первого отражения", ТМФ, 175:2 (2013), 279-299.

[6] V.N. Likhachev, O.I. Shevaleevskii, G.A. Vinogradov, "Quantum dynamics of charge transfer on the one-dimensional lattice: wave packet spreading and recurrence", Chinese Phys. B, 25:1 (2016), 018708.

[7] V. A. Benderskii, E. I. Kats, "Propagating vibrational excitations in molecular chains", Писъма в ЖКЭТФ, 94:6 (2011), 494-499.

[8] В. А. Бендерский, Е. И. Кац, "Распространение возбуждения в длинных одномерных цепочках: переход от регулярной квантовой динамики к стохастической", ЖЭТФ, 143:1 (2013), 5-19.

[9] F. A. B. F. de Moura, R. A. Caetano, B. Santos, "Dynamics of one electron in a nonlinear disordered chain", J. Phys.: Condens. Matter, 24:24 (2012), 245401, 5 pp.

[10] F. Piéchon, "Anomalous diffusion properties of wave packets on quasiperiodic chains", Phys. Rev. Lett., 76:23 (1996), 4372-4375.

[11] A. S. Pikovsky, D. L. Shepelyansky, "Destruction of Anderson localization by a weak nonlinearity", Phys. Rev. Lett., 100:8 (2008), 094101, 4 pp., arXiv: 0708.3315.

[12] S. Flach, D. O. Krimer, Ch. Skokos, "Universal spreading of wave packets in disordered nonlinear systems", Phys. Rev. Lett., 102:2 (2009), 024101, 4 pp.

[13] H. Hatami, C. Danieli, J. D. Bodyfelt, S. Flach, "Quasiperiodic driving of Anderson localized waves in one dimension", Phys. Rev. E, 93:6 (2016), 062205, 9 pp.

[14] B. Huckestein, R. Klesse, "Diffusion and multifractality at the metal-insulator transition", Phil. Magazine B, 77:5 (1998), 1181-1187.

[15] G. S. Ng, T. Kottos, "Wavepacket dynamics of the nonlinear Harper model", Phys. Rev. B, 75:20 (2007), 205120, 5 pp.

[16] R. Ketzmerick, K. Kruse, S. Kraut, T. Geisel, "What determines the spreading of a wave packet?", Phys. Rev. Lett., 79:11 (1997), 1959-1962.

[17] Г. Н. Ватсон, Теория бесселевых функиий, т. 1, ИЛ, М., 1949.

Поступила в редакцию 28.09.2017, после доработки 13.12.2017 